\title{
Complex IT Environments: Ascertaining Information Integrity
}

\author{
Piet J.M. Poos \\ Ernst \& Young EDP Audit \\ Nijenrode University
}

Key words: information integrity, general ledger, client/server, control measures, information architecture

\begin{abstract}
:
In this paper we look at the effects on control measures of the change from mainframe architecture to 2 tier client server to $\mathrm{N}$-tier client server. We will see that the traditional measures in a more or less flat file mainframe environment are no longer sufficient in a large (and complex) $\mathrm{N}$-tier client server environment. We propose middleware solutions that will result in moving completeness controls away from the users and move these to the IT environment. To allow users to effect control over information integrity we propose to use the general ledger as a focal point for intersystem and interprocess reconciliation. This results in a new function in such an organisation: the process controller. He is the owner of the large majority of system interfaces and is responsible for the reliability of interfaces.
\end{abstract}

\section{INTRODUCTION}

Developments in business processes and in IT have both been rapid in recent years. Under the influence of Porter (value chain) [1] and of Hammer \& Champy [2], business processes have been integrated within organisations as well as across organisations. The fast pace of change has led to a shift of emphasis away from mainly financial based management information to anon-financial variety.

IT departments of large organisations have been hard put to keep up with these business changes, which has been one of the reasons for the adoption of client/server applications, initially with a 2-tier client / server architecture, graduating later to an $\mathrm{N}$-tier one. Business demands have led to data warehousing, increased use of ERP packages and the deployment of middleware solutions.

The original version of this chapter was revised: The copyright line was incorrect. This has been corrected. The Erratum to this chapter is available at DOI: 10.1007/978-0-387-35501-6_14 


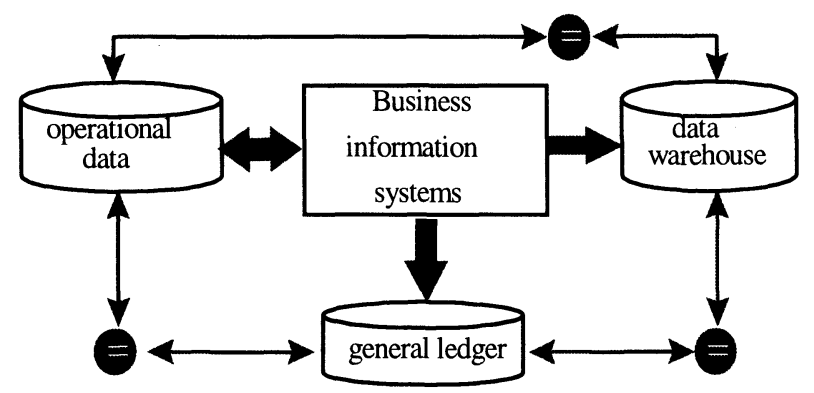

Figure 1: Integrity of redundant data.

The architecture of today's information systems differs from the architecture a decade ago. The mostly batch oriented centralised mainframe processing has gone through major changes. The new component based architecture still has to prove itself in mission critical applications, but the first of these types of systems are at this moment becoming operational. With cheap data storage we see a return of data redundancy (operational data, data warehouse, general ledger). In complex environments, this is already causing integrity problems (see Figure 1. Integrity of redundant data).

The scope of business information systems in large and complex organisations has changed. The systems and procedures that should have supported this change in the back-office however, haven't kept up. Indeed, most of our thinking on the necessary control measures in front-end applications and back office (accounting) systems has remained almost static. This has led to a widening gap between the necessary business- and information integrity controls on the one hand, and the perceived value of integrity controls by managers and systems developers alike on the other.

We will consider the business requirements that drive the increasing reliance on IT. In order to understand the changing control measures, we will look at three different system architectures: mainframe processing, 2-tier client / server architecture and Ntier architecture. For this paper the three architectures can be described as follows:

- Mainframe processing uses terminals or terminal emulation for input. It employs highly concentrated centralised processing, mostly batch oriented. Hardware and operating system architecture includes IBM S-38 (currently IBM AS-400), all varieties of UNIX, Digital Vax, Tandem, IBM S-390 (such as DOS/VSE and VM/MVS) and many others.

- 2-tier client / server splits processing in two. The majority of the application software runs on the client, which typically sends SQL requests to a database on a server. Usually for performance purposes, the server may have some stored procedures in the database. This architecture is also often called fat client because much of the application runs on the client. Server hardware and operating system architecture usually include some variety of UNIX and 
Windows NT, although it is not uncommon to see a mainframe (e.g. IBM S-390 or Open VMS) as a database server. Client operating systems are generally MS/DOS, Windows 3.11, Windows 95 or Windows 98.

- $\quad \mathrm{N}$-tier (3-tier) splits the processing load between clients running a graphical user interface, an application server running the business application software, and a server running the database or a legacy system. In many cases, the business logic can be partitioned over many different servers. This architecture is also referred to as fat server or thin client architecture. Hardware and operating system architectures are similar to 2-tier ones. In addition, $\mathrm{N}$-tier architecture usually employs middleware to connect the various parts of the system together.

In this paper we will explore the changes that have taken place in business information systems and the effects these changes should have had on business process and information integrity controls. We will look at the way these business systems interface with the back office (accounting) systems and will investigate the effects of the introduction of middleware solutions. We will show how the integration of business systems with accounting systems (most notably the general ledger) can improve both business processes and information integrity controls.

For each type of architecture, we assume the same system blocks: maintaining fixed data, input, processing and output (see Figure 2. Basic process model). When talking about maintaining fixed data, we will mainly discuss the way product specifications become available to the user community. This changes rather dramatically, especially in $\mathrm{N}$-tier environments. Where relevant we will also consider general controls in operational IT environments.

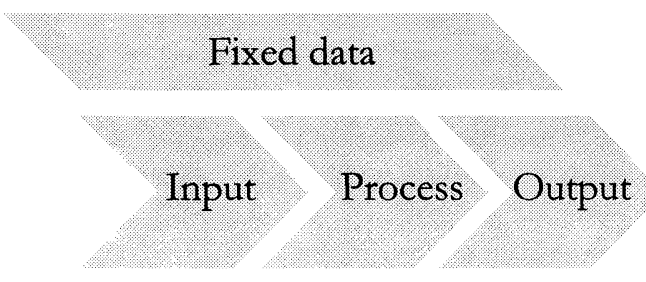

Figure 2. Basic process model.

This paper pursues two different chains of events. First, the development of system architecture from batch oriented processing, through real-time online processing, to the current wave of component-based design. Second, the development of the platforms on which software is deployed (mainframe, 2-tier client / server, N-tier client / server). Although many of the developments in both chains happened at more or less at the same time, the reality has never been as neatly compartmentalised as presented here. Real-time online processing is perfectly possible on a mainframe; indeed, most heavy duty processing still takes place on these systems. It 
is only recently that building mission critical systems on platforms other than a mainframe has become possible.

Information integrity can be defined as the availability of all correct and relevant information at the time and place where it is needed. The scope of this definition is much broader than the usual definition of data integrity. It covers not only reliability but also accessibility, as well as some aspects of effectiveness in the sense of information being delivered on time to the place where it is going to be used. In the course of this paper, the need for this broader definition will become clear.

Business information systems are systems that in financial institutions, for example, hold the transactions with the customer (such as mortgage systems and life insurance systems). The customer is the source and target for, respectively, most input and output.

\section{BUSINESS INFORMATION SYSTEMS [3]}

\subsection{Mainframe processing}

We move back to the end of the seventies, beginning of the eighties, for a closer look at the architecture of business information systems and the influence this architecture had on both programmed and manual control activities. Most data processing was performed within huge mainframe applications that mainly used a batch-oriented architecture. In the banking industry, most branch offices had a network connection with the head office. This was usually intended to retrieve client information (such as account balances) and in some cases to transfer captured data for centralised batch processing (using an FTP-like protocol).

\subsubsection{Maintaining fixed data}

Product information was maintained in various ways. All the distribution channels possessed product information in writing. The sales force was given the ubiquitous rate books to allow them to manually calculate rates and prices, each salesperson being responsible for the maintenance of his or her own rate book. The same products were also hard-coded into the information systems on the centralised computer at the head office. The programs were rigorously tested to verify the correctness of the product specifications used in their design, before being taken into production. For product developers, the product specifications were a static set of formulae for calculating rates, interest and capital. The process surrounding the product (selling, recording, collecting and paying money) was not an issue during product development.

It must be understood that in this period, product specifications were not thought of as a form of fixed data. In system terms, fixed data were interest and exchange rates, and all other data used for more than just one transaction. 
One of the major problems with this architecture concerned the availability of relevant client information. The entire architecture was product based, with each major product (group) having its own system. Automated interrelations between systems were virtually non-existent. The available customer databases were little more than the means to eliminate redundancy in customer address data.

\subsubsection{Input}

At that time, most data capture took place in the branch office. New transactions (such as loan applications) were either written down and transported physically to head office, or they were entered into a system at the branch office to be communicated to the head office for processing by the systems at a later time.

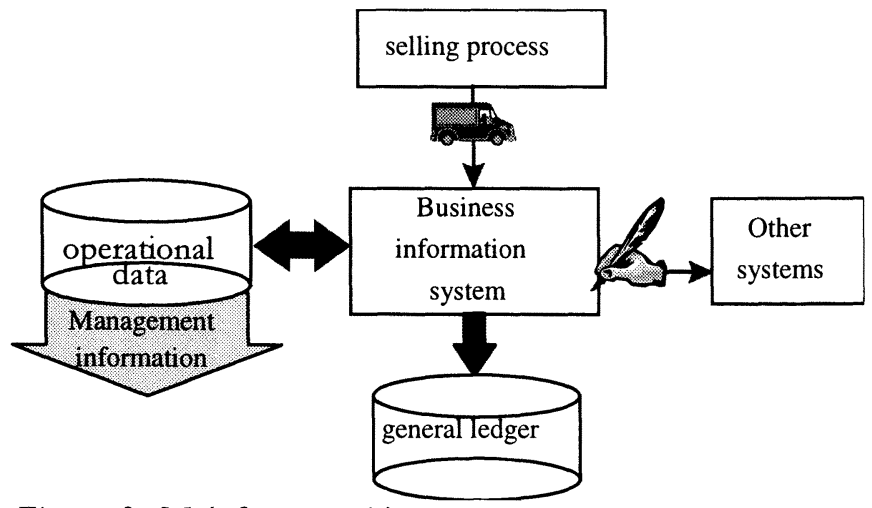

Figure 3. Mainframe architecture.

Although branch offices used some processing systems, these were mainly for generating proposals. These stand-alone systems generally had no connection to the "real" business systems (at head office). Despite all the commotion made at that time about real-time processing, the majority of mainframe systems were very much batch-oriented, with only limited real-time inquiry facilities.

\subsubsection{Processing}

As indicated earlier, most processing was batch oriented. This meant that all input had to be processed at night, as part of "end of day" processing. With the rising number of automated information systems, this put a heavy load on the mainframes.

Just as most data entry was batch oriented, most calculations involving active contracts were based on batch processing. Given the overall performance of mainframes, these calculations (such as interest calculations in a loan system, or renewals in an insurance system) were usually performed on a month-to-month basis. The only exceptions to the rule were contracts being finalised, or expiring during the month. Generally speaking, the number of contract forms was fairly limited, which meant that only a few different types of calculation had to be performed. 


\subsubsection{Output}

In fact, most output from these types of system is generated by processes discussed previously. Mortgage loan contracts, insurance policies, invoices, all these are generated either by processing or when the data is input. There are however some forms of output that merit further discussion:

- Interfaces with other systems. Business processes do not stand alone but are interconnected in numerous ways to other processes. Given the batch-oriented environment, most, if not all, of these interfaces were also on a batch basis. It was not uncommon that these interfaces were almost entirely manual. Of all the interfaces with other business systems, the most notable was the one with the general ledger.

- Management information. At their inception, the majority of systems were able to provide most of the required operational information. Later, more information of a strategic nature was needed. This usually meant that the functions to generate management information were built on top of the operational database.

A large number of systems were developed in this way. Systems that, as we will see, were almost entirely self-managing, and contained all the necessary proof of the integrity of the data they held. As developments continued however, the demands on the systems became greater. Systems had to provide real-time processing and be accessible not only from within the organisation but also by customers. At the same time, the interdependencies between systems became far more numerous. This meant that end-of-day processing became almost unmanageable in its complexity. The time was ripe for a major paradigm shift in architecture design.

\subsection{2-Tier Client / Server Architecture}

Several things were happening simultaneously. On the business side, besides growing third-party access to the network, other developments were going on. Firstly, a shift from product orientation to customer orientation was occurring. As most business systems were highly product oriented, this meant an enormous transformation of the application architecture. Secondly, users were demanding business systems that not only recorded transactions but supported the sales process as well. In addition, the pace was speeding up. The product life cycle in the eighties could be as long as five years; at the start of the nineties, this fell to about eighteen months.

Legacy mainframe applications that had already survived many changes just didn't have the flexibility to endure the overhaul. The pressure on IS departments to deliver the systems that business needed was immense. IS departments, however, were facing huge problems in actually delivering these systems.

On the more technical side, as indicated in the previous paragraph, the mainframe was slowly suffocating from its own complexity. Adding more centralised 
computing power to deal with the extra demand wouldn't solve the problem. Moreover, systems development processes were deemed ineffective by the user community. Given the pressure the IS shops were under, they looked for a replacement for the ineffective waterfall methods.

At the same time, user departments were being stuffed with PCs. Research showed that the computing power of these PCs was only utilised for about 5 to $10 \%$ [4]. The answer was obvious: Let the PC solve the problem. As everyone could attest, building applications for PCs was a lot faster than building them for mainframes. What's more, the unused computing power of the PC could lighten the load for the mainframe. At the same time, the very large mainframes could be downsized and replaced by much smaller departmental systems. 2-Tier client / server computing was born.

\subsubsection{Maintaining fixed data}

The major change brought by client / server computing was the move towards decentralisation. Product information proliferated. Instead of only the paper documentation carried by salespersons and hard-coded into mainframe computer programs, product specifications were now available on multiple computers connected to the network. In large organisations, this meant literally thousands of computers. This proved to be a very mixed blessing at best. Every change to a product specification had to be distributed across the network. Especially where organisations were geographically dispersed, (such as banks), distribution of changed software soon became a major headache. In some cases, the distribution of software alone took several months. This meant that centrally running applications had to be able to cope with several different versions of product specifications. As control over the change process at branch offices was relatively poor, all product applications sent over the network still had to be checked at head office.

Because the availability of customer data was important to users, much effort was put into accessing these data, not only for inquiries, but also for updating purposes. This led to the problem of ownership of customer data, something that proved almost impossible to solve. In most organisations, this resulted in some unhappy compromise or other. It usually meant that some redundancy of data had to be accepted and that not all customer relations could be described in the data model. Even today, pollution of customer data is still one of the major unresolved issues.

\subsubsection{Input}

As indicated earlier, data entry of transactions became more and more a task for the branch office. The architecture of systems to support this task was very different from the old mainframe applications. Mainframe systems were built to process many transactions at the same time (batch processing). The emphasis of these programs was on business logic, and the screens for the input of data were almost incidental. The new architecture was aimed at supporting a user organisation and put the emphasis on ease of data entry. The business logic was very closely linked to the 
input screens (in modern terms: the presentation and business logic tiers cannot be separated from each other). The degree of flexibility of these systems was not very high. Still, the business benefits of this architecture were obvious. During the sales process, all relevant client data could be entered into the system. The simpler transactions could then be finalised immediately and even the contracts could be printed in the branch office. Most of the paperwork was therefore no longer necessary. At head office, this meant that the large departments previously needed to enter new transactions could now be downsized.

\subsubsection{Processing}

The work was not usually performed on the still available centralised mainframe, but on a smaller, dedicated, midrange computer. Calculations on operational data were performed mostly in the same fashion as during the mainframe days. The range of different products had increased and the once-a-month basis for calculations had disappeared. This was made possible by the dedicated computer. As we will see later, the control issues were largely ignored.

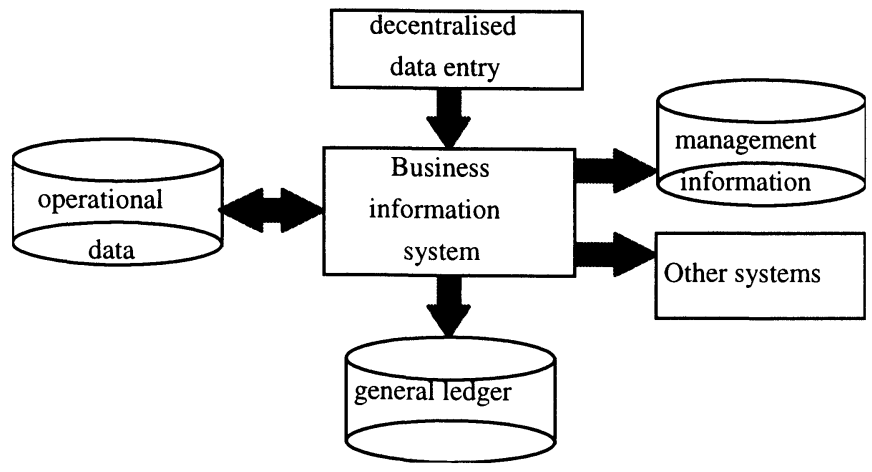

Figure 4. 2-Tier client / server architecture.

\subsubsection{Output}

Interfaces were mostly implemented on a batch-processing basis. Manual interfaces had disappeared, effectively replaced by FTP-like protocols. This meant that complete files were being sent and received. The receiving system had to have a function to check these files. All erroneous transactions had to be put into a suspense file and corrected manually. As the number of these interfaces grew, the time needed to build each one increased. At this time, creating interfaces became the largest task in building a new system. In system maintenance, updating interfaces when the input or output of a system changes represents more than half the work

In the new systems, management information was no longer built on top of operational data. This solution proved to be less than satisfactory. Queries had an adverse impact on performance, especially those that ignored data access paths. 
Also, there was the risk of unauthorised changes. This meant that a separate management information database had to be built, which also needed to be updated.

There are several scenarios for dealing with this problem. The simplest solution is to create a replica of the operational data. Each day, the operational database is replicated, using standard database functions. In many cases though, this is not possible. The MIS data model may be very different from the operational data model. Data access for queries differs from updating access; in other cases, the operational database, for performance purposes, doesn't contain enough historical data. In such cases, the MIS database needs to be updated. This may be done on a regular basis (daily, weekly or even monthly) using a conversion application. It must be clearly understood however, that between updates the content of the MIS database does not correctly reflect the operational data.

All in all, the 2-tier client / server architecture has failed to fulfil its promise. From a business point of view, the architecture has not completely delivered the systems that were needed. Distribution of software and changes to product specifications remain extremely difficult and costly to put into effect. Cost of ownership of these systems has been, and still is, very high. In addition, computers in the branch offices are positively groaning under the weight of all the fat client applications built by the various business units.

Small wonder then at the ever-increasing pace with which other solutions are sought. It must be understood that even today, many 2-tier client /server systems are still being implemented. Most ERP packages are based on this architecture, but most suppliers are working to create a new form.

\subsection{Components and $\mathbf{N}$-tier client / server architecture}

Internet technology is changing the face of front-end business systems at a tremendous speed. Transactions can be executed by almost anyone and at any time. It is not yet a 24-hour economy, but we're getting close. Business systems must be open for everybody, and having a private or closed network is fast becoming impossible, not to say undesirable. In this paper, the Internet security issue won't be addressed. Not because it lacks interest, but because it falls outside the scope of the paper. The business community expects almost instantaneous communications. New opportunities must be translated into products at an unprecedented rate.

\subsubsection{Maintaining fixed data}

The greatest change in maintaining fixed data is in the area of product specifications. Building the systems to sell the products has always been seen as the major bottleneck in the total time to market. In order to drastically reduce the building time, a new answer has been found: product components. 
This approach analyses all products in the product portfolio and breaks them into the smallest possible parts. These very fine-grained parts are then normalised and recombined to form more coarse grained, meaningful components. The result of this exercise is a component model of the product portfolio and consists of not only the data but also the routines to change these data. These product components are not distributed across the network. With $\mathrm{N}$-tier architecture, only the presentation layer is distributed across the network. All business logic software remains centralised, which greatly diminishes distribution time.

\subsubsection{Input}

With the Internet, virtually everybody can enter transactions into systems. There is a genuine need to have real-time processing for new transactions. Save a few isolated cases, most banks and insurance companies do not currently allow the general public to use the Internet to enter business transactions. The issue of foolproof identification and authorisation needs to be resolved first.

Still, the number of sites where banking transactions can be initiated has grown considerably as more sales processes are moving from bank offices to clients' homes and businesses. In the retail sector, of course, it has long been possible to initiate business transactions over the Internet, using credit cards. Here too, the security debate is far from over.

\subsubsection{Processing}

There is little difference in business requirements between processing on 2-tier and $\mathrm{N}$-tier architectures. The number of products has continued to grow and the interval between processing runs is, if anything, even shorter. The main difference is on the technical side. Processing is governed by the components. Each contract needs to be processed by its own components, and. changes to components on active contracts would immediately mean changes to their processing. We need to be certain, therefore, that components remain unchanged.

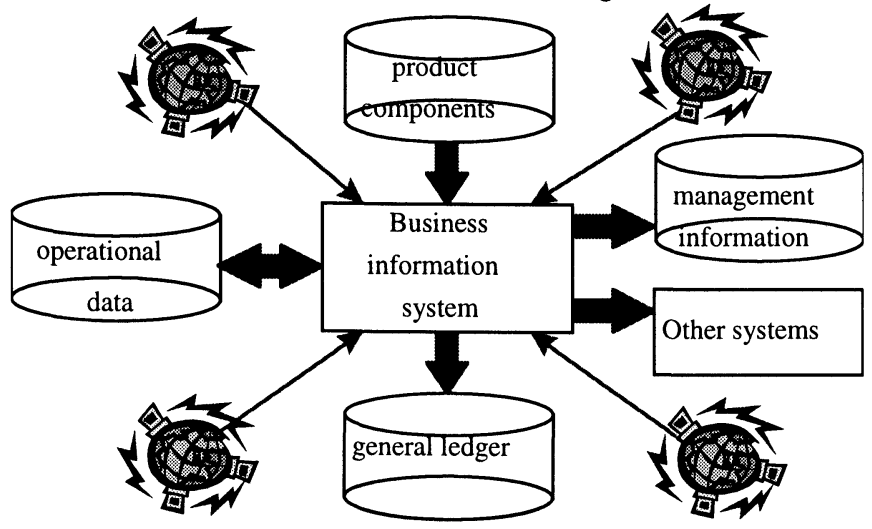

Figure 5. Components and N-Tier architecture. 


\subsubsection{Output}

There is a huge difference between the interfaces in mainframes and 2-tier client / server architecture and the interfaces in $\mathrm{N}$-tier architectures. The difference is not implicit in the architecture itself, but is caused by the business requirements. There has been a shift from batch oriented, seem-manual interfacing to fully automated real-time interfacing.

The real-time character of the interfaces creates additional problems with transactions that are refused by the receiving system. With batch interfaces, these transactions are placed in a suspense file to be sorted out later. This is not a viable option in a real-time environment; different control measures must be employed.

With financial systems in particular, every transaction generates a posting to the general ledger. That in many cases the construction of the general ledger is not capable of processing the generated postings on a real-time basis doesn't change the essentially real-time nature of the interface.

As indicated above, management information is recorded in a separate database. Given the real-time character of the processing, there is no reason why the interface with the management information database should be treated differently from the other interfaces. The cleanest solution is to integrate the update of the MIS with the transaction itself.

With the growing interest in non-financial information, the need to find relationships between data from different systems is growing. This has led (and is still leading) to the consolidation of the various management information databases into one large integrated data warehouse. The route being followed is not different from the development of the management information database, that is, evolving from functions on top of different databases to a single separate database that is first updated using a batch approach, moving later to real-time updating.

\subsection{The future}

It is never easy to predict the future, especially as most developments of the previous paragraph are not yet mature. What we have seen is that systems have been integrated within companies. The next step will be the integration of systems across different enterprises. Some of this integration has already been realised. It used to be called EDI; the modern name is business-to-business e-commerce.

Even today, we need telecommunication systems that provide seamless integration between companies. An example will clarify this. With my (Dutch) mobile telephone, I can call a colleague in Birmingham (UK) from Bordeaux (France). The Dutch company will bill me for this conversation, but needs to pay both the French and the British telephone companies for the use of their infrastructure. The French and the British companies need to be informed they will receive a certain amount of 
money. With the millions of conversations going on, this has to be accomplished using fully automated systems. Imagine what will happen when we can use this same infrastructure to order both a movie on pay-per-view and a pizza, and get billed later by the television company. The telephone company, the television company and the pizza company all want to be paid. This has to be completely transparent to the client who just wants to watch the movie he has ordered, while enjoying his pizza, comfortable in the knowledge that he won't be overcharged for all these transactions.

\section{CONTROL MEASURES [5]}

\subsection{Scope}

At the start of this paper, we introduced the concept of information integrity. In this section we will explore the control measures in the various processing architectures. We will see that the differences in architecture result in different control structures.

Before we start defining control measures, we need to define our control objectives. This is unfortunately not as easy as it sounds. There are two main groups of controls: controls over the business process; and controls over the data processing surrounding the business process. In many cases, there is a large area where these two groups overlap. In this paper we will concentrate on the controls over data processing. This means that our prime control objective is to determine the accessibility of all the correct and relevant information at the place and time it is needed (information integrity).

The are several elements in the definition that are outside the scope of this paper. General controls over the availability of processing (business continuity planning) and general logical access control measures will not be discussed. What will be discussed, however, are the controls over the completeness and correctness of processing, both during processing and afterwards.

\subsection{Maintaining fixed data}

In mainframe processing, product specifications were not seen as fixed data. In processing terms, they were programs. Ascertaining the correctness of these programs was performed by testing. The objective of the tests was to prove that manual calculation of the formulae and electronic processing of transactions produced identical results. In almost all cases, this meant that a test environment separated from the production environment was used. This did not change much with the coming of 2-tier architecture. Product specifications were still seen as programs that needed to be tested. Because of the weaknesses in the control over software distribution, each transaction that reaches the database needs to be checked. Usually this means that the transaction carries an identification of the version of the 
software actually used and is recreated using the correct version. This makes testing fairly difficult and prone to errors.

The component approach to product specification will drastically change the product development process. As indicated earlier, product development concerns itself only with the definition of formulae to calculate, for example, prices, rates and capital. The process of selling and recording product contracts has always been outside the scope of product development, being viewed as part of the domain of systems development.

When using product components, this approach is no longer feasible. Some type of component administrator must be part of the product development team. At a very early stage, this person must translate the first ideas of the team into product components. The fine-tuning of the product will be a process of manipulating the parameter settings of the components, rather that a series of manual what if calculations (using a spreadsheet). Part of product development will be the development of the processes surrounding the product (that is, preparing proposals, entering contracts, changing existing contracts, calculating interest, renewals and expirations). As product development is essentially a creative process, there should be few, if any, restrictions on the initial parameter settings.

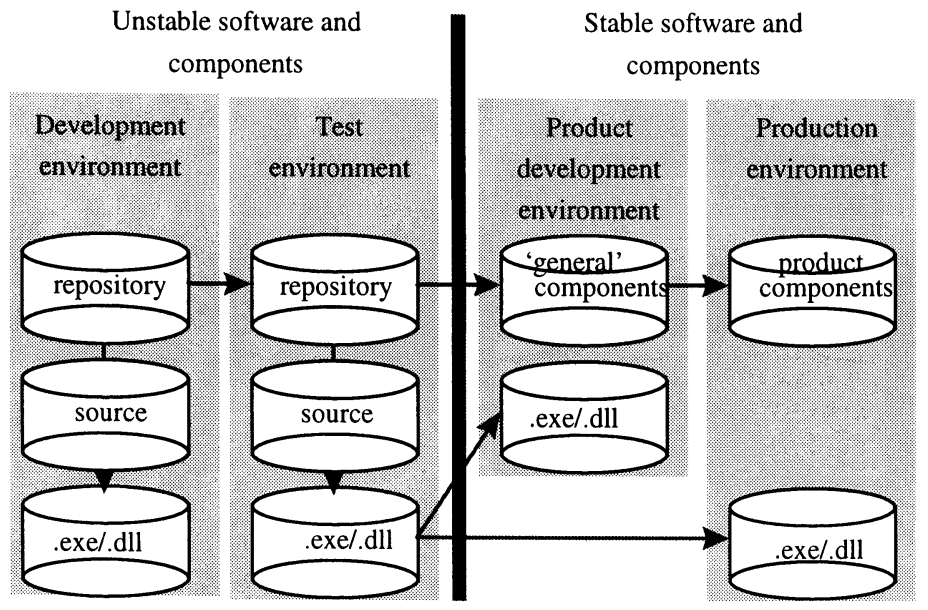

Figure 6. Product Development Environment.

For defining products in this fashion, product development and systems development will become inseparable. When senior management accepts the product, they will also accept the system. This means that the set of parameterised components describing the product needs to be tested before the final decision is made. It is advisable to have clear acceptance criteria for new products waiting to be 
tested. The entire systems development and product development environments need to be equipped to handle this process. At the very least, it means that a separate product development environment must be created (see Figure 6. Product Development Environment).

\subsection{Input}

For the reliability of transaction data, two aspects are of prime importance: correctness and completeness. In both areas the required changes have been obvious.

\section{Correctness}

In mainframe processing, systems relied for correctness on field controls during input. In most literature, "migration of controls" was discussed. This meant that the hard-coded field controls were supposed to be replaced by an active data dictionary/directory system (DD/DS) that would integrate most application-based field controls. In theory, this was correct. In practice, however, the maintenance of the DD/DS was no easy matter. Rapidly changing business rules led to the need for frequent modification of field controls. This was a time consuming procedure at best, since only the database administrator (DBA) had access to the DD/DS, and he or she was swamped with such requests (sometimes even conflicting). The values in the DD/DS became more or less meaningless, useful for correcting only the most obvious errors (in some cases, the interest rate could have value between $0 \%$ and $100 \%)$.

There were some changes in the 2-tier client / server architecture. Most of the tools used to build the systems generated configurations in which most controls were linked to the input screens. The performance of these systems was not very impressive, but given the large number of PCs used, this was not a problem. As said earlier, changing the controls was a problem.

In the component architecture, field controls become part of the product specifications. As such, they are the subject of analysis during product development. At the same time, we see that in most organisations, the sales force is considerably more empowered to makes its own decisions and gets judged much more by results. This means much looser control over input and as an inevitable result, field controls become de-emphasised.

\section{Completeness}

Mainframe processing relied for completeness controls on a set of control equations [6]. This was used in a technical as well as a logical fashion. In this paper, only the logical use will be discussed.

The concept of the set of control equations was derived the flat file environment, where checksums were used to control transaction movements from one flat file to another, primarily in a batch-oriented environment. The concept was translated 
without much difficulty to the database environment. It even afforded extra control because the set itself became part of the data model, as a separate entity. As most systems were still very much batch oriented, the transition to a database environment posed no real problems. For systems that were real-time oriented, batch processing was simulated by defining end-of-day routines for example.

The ultimate purpose of the set of control equations is to reconcile the input to the system with the output in situations where this isn't immediately possible. To achieve the goal, the process is divided into smaller steps, each with its own inputs and outputs. The main idea underlying the set of control equations is the equation:

Opening balance + new transactions - processed transactions $=$ closing balance. In defining the relations, processes that change the state of an entity need to be mapped against the data model. Given the amount of work this entails, it was only performed for the most important (high-risk) entities.

Using a mortgage example, this means that new loan applications are reconciled with the total of payments to customers. To accomplish this the following steps have been designed:

- $\quad$ opening balance of loan applications (to be accepted) + new loan applications accepted loan applications - rejections $=$ closing balance of loan applications (to be accepted);

- opening balance of loan applications (to be processed) + accepted loan applications - processed applications $=$ closing balance of loan applications (to be processed);

- $\quad$ processed applications = payments to customers

- opening balance of outstanding loan applications + payments to customers redemption $=$ closing balance of outstanding loan applications.

This enables the organisation to follow the flow of transactions though its various stages. The closing balances in this set of control equations have to be checked regularly against the total in the mortgage system. If properly designed, the set contains totals (control equations) that are meaningful for the organisation. In the example, every process step can be followed independently.

The transition from mainframe processing to 2-tier client / server architecture didn't go without problems. In theory, the set of control equations could also be deployed in the new real time environment. In practice, serious drawbacks became apparent over time. On the input side of the system, processing was completely real time; there were simply no batch values to compute and track. Each transaction recorded its own details in the appropriate contract database as well as in the "control equations file". The extra control that this afforded was not very great. To put it bluntly, this only served as a way to detect bad programming if the programmer had forgotten to change the totals file. 
Moreover, the set of control equations is most effective only if the individual values (totals) have significance for the organisation. When data entry is decentralised, the total of new transactions can have significance only for the branch office. Since the branch office records only a few transactions each day, other means are used to control the completeness of transactions. The centralised support organisations have been largely dismantled and few departments felt any responsibility for the completeness of new transactions. Testing was mainly used as a preventive measure to guarantee the completeness of input. Unfortunately, systems were often not tested as thoroughly as advisable. (I have experience of a case where contracts for a product were input, but never arrived at the head office. Only after several weeks did someone notice that the production for that particular product was way below budget. All details of input transactions were lost. The cause was a barely tested change in another product specification. The monetary loss was negligible; the loss of image, however, could not even be guessed at.)

The possibly most serious shortcoming has been virtually invisible. In contrast to the traditional mainframe application, a client / server application runs every individual transaction across multiple computer platforms [7]. In the mainframe, a transaction monitor (such as CICS or VMS) guarantees that all transactions are processed. In the multiple platform client / server application such a safeguard is missing. Hence, this preventive measure has to be built for each and every client / server system. In many cases this has been neglected.

To guarantee the completeness of transactions, the traditional approach has become useless. Two main problems need to be addressed. First, given the almost 24 hour / 7 day availability of systems, it becomes almost impossible to simulate an end-of-day process. Even if this were feasible, the value of the additional control afforded by this measure would be negligible. Second, the number of interrelations with other processes and systems has increased.

A transaction in one system usually leads to another transaction in a completely separate system. As the number of relationships increases, the number of interfaces increases even more. In older environments, most of the interfaces were (semi)manual and were essentially bridges between batch processes. In the component and $\mathrm{N}$-tier architectures, the user community wants interfaces to be real time in character as well.

Transactional control can be achieved by deploying middleware, such as a distributed transaction processing monitor (DTPM) or message oriented middleware (MOM). For environments that use a single database system from one vendor, the DBMS can also perform transactional control. Using middleware solves two problems. The completeness of transactions can be guaranteed by the middleware (assuming that the middleware is properly implemented and that the interfaces with the systems it supports are properly built). All faults and irregularities are reported to the middleware system administrator. Unfortunately, these reports are unintelligible for the untrained. This means that the control over the completeness of transactions 
moves irrevocably from the realm of the user community to the domain of systems operations. This has happened before, when control over referential integrity moved from the user to the DBA.

\subsection{Processing}

As we have seen in the mainframe era, each business system is very nearly a selfmanaging entity. Within their own architecture, they carry the proof that operational data are correct. We have also seen that in the 2-tier architecture, there has been an attempt to incorporate the same sort of controls. Given the different processes in client / server systems, especially for entering new transactions, these controls are fairly weak. In the $\mathrm{N}$-tier architecture, we have seen a veritable explosion of possible locations that can initiate transactions. This means that we have to seek another approach for solving the problem. As already observed, keeping track of the completeness of transactions is no real problem. With the correct implementation of the tools, we can be reasonably certain that all new transactions will be processed. The real problem, however, is to obtain a reasonable assurance that the batchprocesses have accessed all active contracts. In the mortgage example, this means that interest and repayments must be calculated for all outstanding loans.

As long as processing takes place on a monthly basis (usual in mainframe processing), the control measure is fairly simple. This is generally a report, generated during processing, that indicates the total number of records that have been processed. It also gives information on the monetary amounts that have been processed (such as total outstanding loans and total calculated interest). This information is reconciled with the set of control equations.

In client / server architectures (both 2-tier and N-tier), the number of processing runs increases dramatically. This means that a processing report is no longer a feasible control measure. We will have to fall back on a considerably more indirect monthly report that indicates essentially the same information. As it is not generated during processing, such a report is generally considered a much weaker control measure. If the report can be reconciled with other information sources (like the generated interest posted to the general ledger) this strengthens it as a control measure.

\section{5 .}

\section{Output}

As indicated above, interfaces in the $\mathrm{N}$-tier environment have become fully automated. This worsens the cross-platform problems in maintaining transactional control. Fortunately, with modern middleware tools these problems can be solved. There are several possible scenarios depending on the sort of cross-platform control that is needed.

- Synchronous communication mechanisms such as request-response or conversational ones that use two-phase commit. This is a communication method in which the middleware checks whether or not each system that has to process 
the transaction is actually ready to do so. If during processing, one of the systems fail to acknowledge the transaction, the complete transaction is rolled back and refused. It really is an all or nothing approach. The main advantage is the very direct control. All systems involved in the transaction process the data simultaneously, with no timing difference to speak of. There are three main disadvantages.

1. Two-phase commit is very network intensive and uses a lot of bandwidth. When high performance is demanded, this can be a drawback.

2. In large environments with many interfaces, two-phase commit may become very complex and difficult to maintain.

3. Two-phase commit expects systems to use recoverable resources (loosely called XA compliant [8]). Unfortunately this is not always the case.

These last two disadvantages are a real bottleneck to implementing middleware in existing organisations.

- Publish and subscribe. This is an asynchronous way of communicating in which the sending system "fires" the transaction at the middleware and can promptly "forget" all about it (fire and forget). The middleware knows which systems will use the transaction and takes care of all the distribution issues. The sending system does not need to know which systems subscribe to the data, since the middleware handles everything. Subscribing also has several options. The middleware can ship a data item the moment it is received, or the receiving systems might have a routine that regularly checks to see if data are available. This is a type of interface that is intrinsically much simpler than two-phase commit and uses a lot less bandwidth. On the other hand, if input from the receiving system is necessary to complete a transaction, a more conversational way of interfacing is needed. Using publish and subscribe can also lead to different processing times in different systems which may in turn lead to apparent irregularities caused purely by timing differences.

With these forms of transactional control, we can have a reasonable -technicalassurance that all initiated transactions will be processed. A large organisation, however, is not a closed system. The need for manual correction in such environments will remain. When the number of transactions that are executed by other systems rises, it becomes more important that the initiating transaction and these other transactions are reconciled fast. In every organisation that processes large volumes of transactions, mistakes will be made, and in financial institutions, there is always the possibility of fraud. This means that anomalies must be quickly detected and corrected.

Reconciling the transaction flows from different processes has always been an important issue in Dutch theory on business process analysis. In the mortgage example above, it was almost mandatory that the total of finalised mortgages should equal the total paid loan applications. Given the timing differences that exist between the various processes in an $\mathrm{N}$-tier environment, it becomes almost impossible to prepare an overall reconciliation. This means that reconciliation has to 
be carried out in detail, in other words, each executed payment as processed by the payment system must be reconciled with a finalised loan from the mortgage system. With the large number of transactions to be processed, the reconciliation will have to be performed automatically. Anomalies will have to be reported and followed up immediately. In many cases, the number of reconciliation's will be very large. This means that all transactions to be reconciled will have to be collected in a system for performing reconciliation's and reporting anomalies.

As far as management information goes, there are three issues of interest.

1. The content of management information.

As indicated earlier, we have seen that alongside technical changes in architecture, there have been changes on the business side as well. In controlling business processes, there was a heavy emphasis on thoroughly checking each transaction as it was entered. What we see now is that salespeople are allowed more latitude in completing a transaction, making it more difficult to check each one. As a result, the focus of control over the business process moves from preventive detailed measures embedded in the input functions to overall repressive information about completed transactions. This information will probably be derived from the management information database. This means that the management information database is playing an ever more important role in controlling business processes. In this paper, we will not discuss the content of management information any further.

\section{The relevance of the information derived from the MIS database.}

The purpose of the MIS database is to supply the organisation with information, generally on a regular basis. Much of this is based, one way or another, on performance indicators. A minor part of the information from the MIS database (but nonetheless a very meaningful part) comes in the form of ad hoc queries. The main problem is how to control the quality of these queries. Standardised queries pose few problems in this respect, since they can be and are tested. In many cases, however, ad hoc queries are often built by the people submitting them, that is, non IS personnel. Often, these queries are not tested at all, just given a cursory glance, with a "this looks what I need". Unfortunately, there is no way to control this process. Personnel can be trained, we can have decentralised "information desks" whose sole task is to build queries, but there is no cure for stupidity and ignorance.

\section{The completeness and correctness of the data in the MIS database.}

Since the MIS database is intended to reflect operational data, we must have tools to guarantee the completeness and correctness of the MIS database. The simplest solution is to reconcile the data in the MIS database with the operational data. This is not as easy as it sounds. We have seen that over the years, several scenarios have been developed for filling the MIS database. Many of these scenarios allow a time lag between the update of the operational systems and the update of the MIS database. In reconciling these two sets of data, we must allow for any time lag. 
The retention period for data in the operational systems might be quite different from the retention period in the MIS database. In a mortgage system, this wouldn't pose any problems. There are many other, much more volatile processes, where the retention period would be an issue. In my experience, a foreign exchange system in a major bank only retains expired contracts for about three months; a longer period would put too heavy a stress on the performance of the system. Reconciling active contract balances with those in the operational system would be possible here; reconciling the generated income over the current annual would soon be impossible however.

In many cases, the data model of the MIS database differs significantly from the data model of the operational systems. This in itself makes reconciliation difficult. The problem increases when the MIS database starts to contain data from more than one system. It becomes clear that reconciling an MIS database with the operational data is not a simple matter. It also is clear that, given the importance of the MIS database, some form of control is absolutely essential. The general ledger system often contains much of the financial information that originated from the operational systems. A solution would therefore be to use the general ledger as a source for validating the MIS database.

\subsection{The framework}

So far, we have been discussing the problems that have to be faced in designing Ntier systems. We have seen that we need to have transactional control over the complete data processing (maintaining fixed data, input, processing, output). When interfacing with systems, especially if the interface generates transactions to be processed by other systems, we need a way to reconcile (logically) the generated and processed transactions. What we must realise, is that such a combination of systems are not a stable and closed environment.

Changes will be made continuously. We have seen the need for continuous product development, and we have looked at the systems that will make this possible. At the same time, legacy systems will be replaced by new N-tier systems. Furthermore, maintenance of existing systems will continue.

In any large organisation, there is a need for human intervention. Particularly in situations where transactions are generated by the public, the likelihood of errors and incomplete transactions is great. It is up to the organisation to refine and massage the data to make further processing possible. With the systems integration that has taken place until now, these manual corrections are likely to have effects in other systems as well.

What we need is a tool to keep control over, ideally, the entire structure of business processes, reconciliation systems and MIS databases. The funny thing is that we already have this tool (see Figure 7. The general ledger as a tool for process 
control). For generations, we have been using the general ledger as the repository of accumulated (financial) information. It was in fact the first management accounting information system [9].

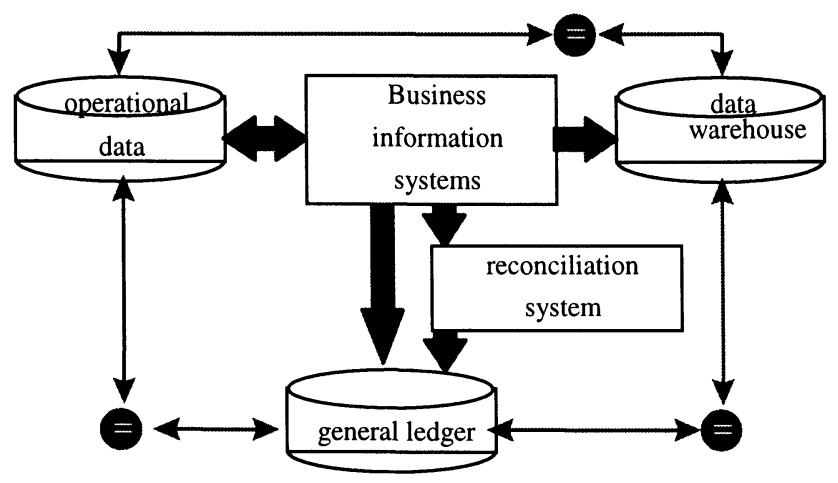

Figure 7. The general ledger as a tool for process control.

There are several reasons why the use of the general ledger has declined. The primary reason is that the general ledger is no longer seen as the primary source of management information. This role has been taken over by the MIS database. The general ledger has become simply a tool to generate the annual accounts. The secondary reason is that many organisations have started to use a multitude of different systems, many of which are standard applications. These packages generally have some sort of general ledger, which means these organisations don't have one general ledger system, but have several of them.

This might also be the reason that the use of the general ledger as a tool for process control rather than financial control has been overlooked. If used properly, the financial results of all transactions will have to find their way into the general ledger. This means that one way or another the balance of the general ledger account can be reconciled with the data in the operational systems (such as the closing balance of outstanding mortgages). The same goes for generated income. This too can be reconciled on a monthly basis with the operational systems. As the retention period of data in the general ledger can much longer than the retention period of the operational systems, the general ledger can also be used to control the integrity of the MIS database.

As we have already seen, the total environment is by no means a closed system and we need to properly reconcile linked transactions that have been processed by different systems. We have introduced the concept of a reconciliation system to perform the task. This system may or may not be an integral part of the general ledger. 
The general ledger and the reconciliation system together become the principal tools for controlling a complex integrated environment. The owner of these tools should be a new function in such an environment: the process controller. The primary task of this function is to ascertain information integrity, with the task employing several different means:

- controlling the interfaces between systems and verifying that these interfaces work correctly;

- checking that the balances of active contracts in the various systems can be reconciled (closing balance of active loans in the mortgage system = outstanding loans in the MIS database = the outstanding loans in the general ledger);

- defining the - mainly logical - standards with which systems must comply to be allowed to interface with other systems.

Up until now, these tasks have been performed by the financial controller because financial and process controls seem to be very closely related. With the increased dynamism of the business environment and the in-depth knowledge required for business processes and IT solutions, it is becoming more and more difficult to combine both functions in one person.

It must be stressed that process control is not an IS function, but a job for the user community. Although process control will be instrumental in defining the requirements for interfaces, the main area of work lies in the operational systems.

\section{THE GENERAL LEDGER}

So far, we have only seen some tantalising small bits and pieces from the general ledger. Now it's time to take a closer look. Over the years, the general ledger has grown into a general-purpose tool, mainly for financial control. The following functions are fairly commonplace in most organisations:

- Maintaining the relationships between assets, liabilities, costs and income. Historically, this is the function that the general ledger started with. It is also this function that would help us most in regaining process control.

- Support for (financial) reporting. As said earlier, the general ledger was the very first repository for (financial) management information. Over time, this has become one of its most important functions. Many of the other functions follow directly from the fact that the general ledger contains information about most other processes in the organisation.

- Recording and safeguarding accounts payable and accounts receivable, both in detail and in total. In principle, neither accounts receivable nor accounts payable are part of the general ledger. Obviously, the total balances of accounts payable and receivable will be contained in the general ledger; the details should be contained alongside the business systems. This holds true for all subledgers. 
- Suspense accounts. It has always been a function of the general ledger to reconcile transactions from various processes. This usually concerns processes that are very closely related to the general ledger. Depending on the scope and number of transactions, this function can be relegated to a subledger (since, for example, most banks use a separate system to reconcile interbank transactions). In our framework this function becomes the second cornerstone of process control.

- Recording intercompany accounts. Most general ledger systems have the functionality to record and reconcile intercompany accounts; this could be treated as a subledger of the general ledger.

- Support consolidation. Most larger general ledger systems have the functionality to support consolidation. In most complex organisations, consolidation will not be supported by the general ledger but by a separate system.

- Recording budget utilisation. Again, most larger general ledger systems have the functionality to support the budget process. In most complex organisations, budgeting will not be supported by the general ledger but by a separate system.

- Recording tax liabilities. In most cases, this concerns the liability for various kinds of taxation such as VAT and sales tax, taxes on wages and salaries, social security contributions, company tax and others that are recorded directly in the general ledger. Given the many processes involved (or in some cases, the lack of processes), the most efficient solution is often to hold this information within the general ledger.

Over the years, and with the extra possibilities afforded by IT, the general ledger has become a veritable cornucopia of functions and related information. It is just this stacking up of functions that gives the general ledger the character of a Swiss army knife (you can do almost everything with it, but you can't do anything really well). Cutting away the superfluous functionality could greatly enhance the remaining functions. This would mean a rigid division between the reporting and the control functions.

The reporting functions become part of the data warehouse. This would be possible because process control also guarantees the information integrity of the data warehouse. In most cases the data warehouse already contains most of the detailed information necessary to prepare adequate financial and management reports.

In our framework, the control functions remain the purpose of the general ledger. However, it must be realised that process control may require a completely different organisation of both the general ledger and the chart of accounts. Given the legislation surrounding the general ledger in some countries, the impact of this may be wider than would appear at first sight. 


\section{CONCLUSIONS}

We have seen that IT has become an integral part of every aspect of business processes. Even in the most decentralised processes (such as the sales process in people's homes), it is now common to use computers. In this decade, computerised systems have become more integrated as well. The users of these systems now expect disparate systems to work seamlessly together. For the foreseeable future, we can expect that integration within organisations will soon give way to integration across organisations. In developments concerning business-to-business e-commerce, this has already started.

While the front-office has been moving forward at a rapid pace, this has not been emulated by back-office systems and control measures. The change from mainframe computing to 2-tier client / server systems has led to a structural decrease of control over information integrity. Neither tools nor procedures are adequate to the demands of an inherently more complex processing paradigm. With the coming switch to $\mathrm{N}$ tier client / server processing, we will soon have the tools to implement transaction control in a complex environment, on a technical level. However, most organisations still don't have the procedures in place that form an integral part of overall process control.

The general ledger and a reconciliation system can together become the tools to bring about process control: the general ledger to reconcile balances and results over the various systems; the reconciliation system to reconcile transactions across systems. As so many parts of the organisation are involved, it will become necessary to further develop a function that controls the integrity of interprocess and intersystem interfaces: the process controller. The primary task of this function is to verify that all interfaces between the various systems are working correctly and completely. The correctness and completeness of interfaces must be monitored constantly. With the dynamism and open character of modern business environments, a measure that only enhances prevention cannot give the assurance that is needed.

As integration continues, there will be a growing need for a function like the one just described. We can be sure that in any large-scale e-commerce environment, this function is going to be an absolute necessity. It will free financial controllers to perform their tasks without the problems of the integrity of the information they have to use in their work.

\section{REFERENCES}

1. Competitive Advantage: Creating and Sustaining Superior Performance; Michael E. Porter, ISBN: 0684841460. 
2. Rengineering the Corporation: A Manifesto for Business Revolution; Michael Hammer, James Champy; ISBN: 088730687X.

3. Most of the information comes from:

- Client / Server Survival Guide; Robert Orfali, Dan Harkey, Jeri Edwards, ISBN: 047-31615-6.

- 3 Tier Client / Server At Work; Jeri Edwards; ISBN: 0471-31502-8.

- The Tuxedo ${ }^{\mathrm{TM}}$ system; Juan M. Andrade, Mark T. Carges, Terence J. Dwyer, Stephen D. Felts; ISBN: 0-201-63493-7.

4. Gartner Research Group.

5. Mainframe controls are based on "Systems Auditability and Control, part: Control Practices" The Institute of Internal Auditors. ISBN: 0-89413-051-X; $9^{\text {th }}$ printing 1984

6. In Dutch: netwerk van controletotalen.

7. Client / Server: de strijd tegen de complexiteit (Leo Klaver, Informatie Management, mei 1998).

8. e.g. Open Group X/Open OSI/TP standard, CICS 2-Phase Commit protocol, OMG Object Transaction Service

9. Relevance lost: the rise and fall of management accounting. H. Thomas Johnson and Robert S. Kaplan; ISBN: 0-87584-254-2). 